\title{
The BINA collaboration: science at the Royal Observatory of Belgium
}

\author{
Peter De Cat*, Jan Cuypers ${ }^{\dagger}$, Ronny Blomme, Yves Frémat, \\ Martin Groenewegen, Patricia Lampens, Alex Lobel, Thierry Pauwels, \\ Griet Van de Steene, Peter van Hoof
}

Royal Observatory of Belgium, Ringlaan 3, 1180 Brussels, Belgium

\begin{abstract}
The Belgo-Indian Network for Astronomy and Astrophysics (BINA) is a collaboration between Indian and Belgian astronomical institutes with the main aim to optimize the scientific output of the IndoBelgian telescopes, being the 4.0-m International Liquid Mirror Telescope and the 3.6-m Devasthal Optical Telescope. These new facilities are both located at the Devasthal Observatory near Nainital, India. In this contribution, we introduce projects that are of scientific interest for colleagues of the department "Astronomy and Astrophysics" of the Royal Observatory of Belgium (ROB). It serves as an invitation for Indian astronomers to participate. We highlight how these projects could benefit from observations with the Indo-Belgian telescopes by using instruments from the first-generation (currently offered) and/or the next-generation (development or design phase). We show that, from an ROB point-of-view, the BINA would be the most successful if the 3.6-m DOT would be equipped with an efficient optical high-resolution spectrograph.
\end{abstract}

\section{Introduction}

The Royal Observatory of Belgium (ROB) is one of the three Federal Scientific Institutes located at the "Space Pole" site in southern part of Brussels (Ringlaan 3, 1180 Brussels, Belgium). It is subdivided into five departments: "Reference Systems and Planetology" (OD1), "Seismology and Gravimetry" (OD2), "Astronomy and Astrophysics" (OD3), "Solar Physics and Space Weather" (OD4), and "Planetarium" (OD5). This contribution is presented by members of OD3 (Fig 1).

In Section 2, the science that is being done at OD3 is introduced in the form of projects. They serve as requests for collaborations within BINA. We therefore show how these projects could benefit from ground-based observations with the Indo-Belgian telescopes at the Devasthal Observatory, being the 4.0-m International Liquid Mirror Telescope (ILMT) and the 3.6-m Devasthal Optical Telescope (DоT). For the 3.6-m Dot, three instruments will be offered from the first observation seasons onwards:

- an optical imager (spectral range: optical, 360-1000 nm; field-of-view: square of 7x7 arcmin on

\footnotetext{
*e-mail: Peter.DeCat@oma.be

${ }^{\dagger}$ Sadly, Jan Cuypers, head of the department "Astronomy and Astrophysics", suddenly died on 28/02/2017 at the age of 60 years. He is missed a lot by his colleagues and far beyond. We express our most sincere condolences to his family and dedicate this paper to him.
} 


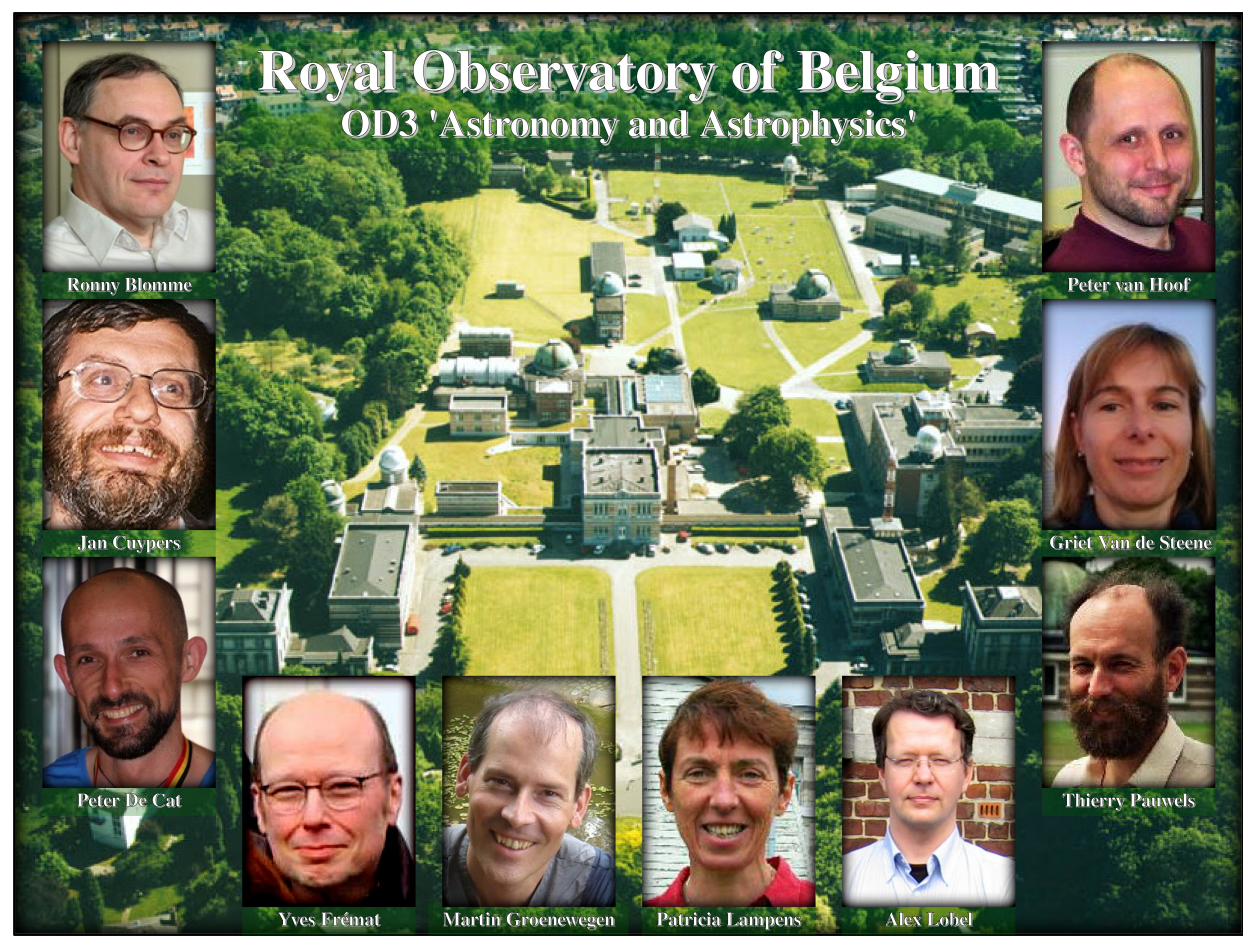

Figure 1: Presentation of the department "Astronony and Astrophysics" (OD3) of the Royal Observatory of Belgium. Pictures of the staff and post-doctoral researchers are given on top of a view on the site where the Royal Observatory of Belgium is located (Ringlaan 3, 1180 Brussels, Belgium).

sky; photometry: broadband Johnson-Cousins $U, B, V, R, I+u, g, r, i, z$ SDSS filters),

- FOSC (spectral range: optical, 350-900 nm; field-of-view: square of 14x14 arcmin on sky; photometry: broadband $u, g, r, i, z$ SDSS filters + narrowband filters centered at 492,661,673,683 nm; spectroscopy: spectral resolution $R$ from 250 to 4000; Omar et al., 2012), and

- TIRCAM2 (spectral range: infrared 1-5 $\mu \mathrm{m}$; field-of-view: circular with 1.14 arcmin diameter on sky; photometry: broadband $J, K$ filters + narrowband $H_{2}, B r_{\gamma}, K_{\text {cont }}, P A H, n b L$ filters +1 block disk; Naik et al., 2012).

We indicate how these first-generation instruments and some of the next-generation instruments, that are under development or in the design phase, could help in achieving the scientific goals of the ROB.

\section{Science@ROB}

\subsection{Follow-up for asteroseismic space targets}

The main interest of P. De Cat (Peter.DeCat@oma.be) lies in asteroseismic studies of pulsating stars on or near the main-sequence. It concerns both gravity mode pulsators (slowly pulsating B stars and $\gamma$ Doradus stars; pulsation frequencies of the order of days) and pressure mode pulsators ( $\beta$ Cephei stars and $\delta$ Scuti stars; pulsation frequencies of the order of hours).

The way a star can pulsate is uniquely determined by its composition and the internal physical processes. In asteroseismology, the observed pulsation modes are used to gather information of the stellar interior in an indirect way and hence to improve our knowledge of stellar evolution. A successful asteroseismic study requires the observation of a significant number of well identified modes (to confront them with those predicted in theoretical models) and the knowledge of accurate stellar parameters (to know which models are appropriate for the studied star). For the detection of the 
pulsation modes and the determination of their frequencies, time series of high-quality photometric (brightness variations) and/or spectroscopic (line-profile variations) data with a sufficiently long time base are needed. For the identification of the pulsation modes, we can use methods based on multicolor photometry (determination of degree $\ell$; Dupret et al. 2003) and/or high-resolution spectroscopy (determination of degree $\ell$, azimuthal number $m$ and other parameters; Briquet \& Aerts 2003, Zima 2006). To derive the stellar parameters $\left(T_{\text {eff }}, \log g\right.$, metallicity, abundances, $\left.v \sin i\right)$, a single highquality, high-resolution spectrum is already sufficient. Several such spectra can also be used to derive the radial velocity and hence to check for multiplicity and/or cluster membership.

In the last decade, space-based observations from missions like Most, CoRoT, Kepler/K2, and BRITE have already proven their usefulness for the detection of many low amplitude pulsation modes. We still have some high hopes for (future) space-missions like GAIA, TESS, and PLATO. As most of these missions only provide single-color photometry, we still have to rely on ground-based followup observations to assemble all the ingredients for asteroseismology. At the ROB, we frequently make use of the HERMES spectrograph attached to the 1.2-m MERCATOR telescope (Roque de Los Muchachos Observatory, La Palma, Canary Islands) to acquire spectra with a resolution $R=85000$ (Raskin et al. 2011). Unfortunately, many of the most interesting asteroseismic space targets are too faint for this facility.

Long term access to the 3.6-m DoT telescope could help the ground-based follow-up for asteroseismic space targets in two different ways:

- Multi-color photometry (optical imager/FOSC): Several filters are offered for both the optical imager and FosC. They are appropriate to gather time series for faint targets to get restrictions on the degree $\ell$ of their pulsation modes based on the amplitude ratios and phase differences in different colors.

- High-resolution spectroscopy (HERMES-like): With an efficient high-resolution spectrograph, we could select fainter asteroseismic space targets for detailed studies of line-profile variations. It would allow to perform a full identification of their pulsation modes with a sufficiently high amplitude. This type of study requires spectra with a signal-to-noise ratio (SNR) of at least $\sim 150$. With HERMES, this can only be achieved with exposure times shorter than $\sim 5 \%$ of the observed pulsation periods for targets with $V$ up to $\sim 8-9$ mag.

\subsection{Projects with the HERMES spectrograph}

Dr P. Lampens (email: Patricia.Lampens@oma.be) is currently interested in research projects involving binarity and stellar pulsation. The aim of these projects is to improve the understanding of stellar physics for stars of intermediate spectral type and mass which are located at the intersection of the main sequence and the classical $\delta \mathrm{Scuti} / \gamma$ Dor instability strips. In this region, a rich diversity of observed phenomena exists related to stellar pulsation, rotation, convection, diffusion and chemical composition, all of which are also under the influence of binarity/multiplicity and magnetic fields.

For the project "Spectroscopic Survey of Candidate Hybrid A/F-type Stars from the Kepler Mission", we are collecting multiple high-resolution spectra with HERMES for a sample of brighter candidate hybrid A/F-type pulsators discovered by the space mission Kepler. We extract the radial velocities, projected rotational velocities, fundamental atmospheric parameters and provide a classification based on the appearance of the cross-correlation profiles and the behaviour of the radial velocities with time. We provide improved fundamental parameters for all observed targets and aim to determine the fraction of spectroscopic systems with the purpose to investigate the cause of the low frequencies that affect the light curves of these candidate hybrid pulsators. This study is performed in collaboration with Drs. Á. Sódor, M. Skarka and Zs. Bognár (Konkoly Observatory, Budapest, Hungary). First results are presented by Lampens et al. (2015), Lampens et al. (to appear in A\&A), and Lampens et 
al. (these proceedings).

In "Orbital Parameters of Selected Eclipsing Binaries with Short-Period Pulsations", we collected multiple high-resolution spectra with HERMES of carefully selected oscillating Algol-type binary systems (named 'oEA stars') in order to construct precise component radial velocity curves. The goal is to determine accurate absolute parameters of these oscillating binary systems as an essential step towards a correct understanding of the pulsation properties in the presence of mass accretion and/or tidal effects in close binary systems. We share this research interest with Dr D. Mkrtichian (National Astronomical Research Institute Thailand, Chiang Mai, Thailand; e.g. Lampens et al. 2011).

"Binarity and Magnetic Interactions in various Classes of Stars" (BinaMIcS) is a large international project lead by the Institut de Planétologie et d'Astrophysique de Grenoble and the Observatoire de Paris. It studies the interplay between stellar magnetic fields and binarity from an observational as well as a theoretical point-of-view (http: / / binamics. lesia.obspm. fr/the-project/). The spectra are acquired with telescopes equipped with high-resolution spectropolarimeters and located at French and international observatories. In collaboration with Dr C. Neiner (LESIA, Observatoire de Paris, France), a detailed study of the time series spectra of an F-type binary system applying the technique of spectra disentangling is presently on-going (Neiner \& Lampens 2015).

For such detailed stellar physics problems, the 3.6-m DOT equipped with suitable instrumentation is a very promising instrument. Ideally, the following techniques should be offered:

- High-resolution spectroscopy (HERMES-like): Using the Devasthal Optical Telescope equipped with an efficient high-resolution spectrograph, our spectroscopic survey could be easily extended to a larger sample of fainter candidate hybrid stars of the Kepler mission. It would also become more easy to acquire spectra with a higher SNR for a given magnitude limit. Additionally, detailed spectroscopic studies based on high-resolution spectra are needed for in-depth studies of different classes of main-sequence pulsators and the new hybrid pulsating classes (cf. Section 2.1).

- Fast multi-color photometry (optical imager/FOSC): When confronted to binary systems which have pulsating components, the determination of the fundamental stellar properties of the components based on an accurate modelling of the system can provide strict constraints on the pulsational properties of the pulsating component(s). In the very important case of eclipsing systems with pulsating components, fast multi-color photometry during the (primary) eclipses can procure significant insight for mode identification purposes (using the eclipse phenomenon as a spatial filter to resolve the pulsations across the stellar surface of the pulsator; e.g. Gamarova et al. 2003).

- Spectropolarimetry: There is also a need for spectropolarimetry in order to check for the presence of brightness or chemical inhomogeneities on the surfaces of the candidate hybrid stars which may be related to magnetic activity.

\subsection{Classical variable stars as distance indicators}

Essentially all stars are variable if studied long enough and with sufficient precision. M.A.T. Groenewegen (Martin.Groenewegen@oma.be) focusses on the classical periodic variable star classes of RR Lyrae (RRL), $\delta$ and Type-II Cepheids (T2C), and long period variables (LPVs), mostly the Mira variables which have the largest pulsation amplitude. All these classes of classical periodic variable stars follow period-luminosty (PL)-relations that can be used, when calibrated, to derive distances to individual objects. This makes them usefull to study the structure of populations in external Galaxies and in our Milky Way (with an increasing number of accurate parallaxes in subsequent Gaia data releases).

The small dispersion in the PL-relation in the classical Cepheids makes them the primary calibrator in the distance ladder, and ultimately in determining the Hubble constant $H_{0}$. Riess et al. (2016) find $H_{0}=73.24 \pm 1.74 \mathrm{~km} / \mathrm{s} / \mathrm{Mpc}$ by using locally calibrated Cepheids to determine the brightness of 
Type-I supernovae (SNIa) in two dozen galaxies, and then measure the distance to SNIa in the Hubble flow. This value differs by more than $3 \sigma$ from the $H_{0}=66.93 \pm 0.62 \mathrm{~km} / \mathrm{s} / \mathrm{Mpc}$ determined by the Planck mission (Planck Collaboration et al. 2016). Whether this discrepancy is real is of obvious importance. It requires that all steps in the stellar distance ladder are investigated and improved. This also includes the zeropoint calibration of the Cepheid PL relation. In this line, the Carnegie-Chicago Hubble Program (Beaton et al. 2016) aims at a 3 per cent measurement of $H_{0}$ using alternative methods to the traditional Cepheid distance scale. They aim to establish a completely independent route to the Hubble constant using RRL variables and the tip of the red giant branch (TRGB). Also this requires a reassesment of the RRL PL relation. T2C could also be of interest in this. They are thought to be low-mass stars of low-metallitiy. They are less numerous than RRL, but brighter. Recently Groenewegen \& Jurkovic (2017a,b) studied the 300 T2C discovered in the OGLE-III survey of the Magellanic Clouds (MCs), and derived the period - bolometric luminosity relation.

One important aspect of PL relations is whether they depend on metallicity, or not. The effect is likely to be small, especially when going to the infrared (Storm et al. 2011, Groenewegen 2013), but nevertheless needs to be quantified if a $1 \%$ accuracy in determining $H_{0}$ is the ultimate goal. M.A.T. Groenewegen has been involved in collaborations that determined abundances in Cepheids (Romaniello et al. 2005, Pedicelli et al. 2010). A related aspect is that individual distances to Cepheids or RRL are sometimes required that are outside direct parallax measurements. The BaadeWesselink (BW) method is such a tool (Storm et al. 2011, Groenewegen 2013 for applications to the MCs). To apply it, both lightcurves and radial velocity data are needed. They can be used to relate the variation in physical radius (through integration of the radial velocity curve) to the variation in angular diameter (by using a surface-brightness relation to link color to angular diameter) and thus derive the distance.

The research on classical variable stars could be expanded with observations from the 3.6-m DoT if the following instruments are attached:

- Infrared camera (TIRCAM2): The strength of the T2C, classical Cepheid, RRL, and Mira PL relation lies in the infrared, where reddening is less and the scatter is intrinsically small. Observations in the infrared are therefore crucial, either for a few epochs to accurately determine the mean magnitude (for variables with known period) or for monitoring to accurately determine the lightcurve needed for the BW method.

- High-resolution spectroscopy (HERMES-like): An efficient high-resolution spectrograph would allow to determine the abundance and metallicity of individual Cepheids (in the Milky Way and possibly in extragalactic systems) and to perform radial velocity studies. Apart from being requirements for the BW method, they would also allow to study the effect of binarity.

\subsection{Spectroscopic monitoring of hypergiants}

A. Lobel (Alex.Lobel@ oma.be) is studying several types of hypergiants.

Cool hypergiants $\left(\mathrm{Ia}^{+}\right.$or Ia0) are rare supergiant stars with $T_{\text {eff }}$ below $\sim 10000 \mathrm{~K}$. They are physically different from supergiants of luminosity class Ia. The $\mathrm{Ia}^{+}$designation does not mean that they are always more luminous, but they differ in their spectral properties. Hypergiants show one or more broad emission components in the Balmer $\mathrm{H} \alpha$ line profile, a signature of an extended atmosphere or of a relatively large mass loss rate (De Jager 1998). The absorption lines of cool hypergiants are significantly broader than those of the Ia supergiants of similar spectral type and luminosity. $\rho$ Cas and HR 8752 are bright prototype stars of the yellow hypergiants (YHG, F-G Ia ${ }^{+}$). They are among the most massive $\left(20-40 \mathrm{M}_{\odot}\right)$ cool stars presently known. They are thought to evolve fast on blue loops in the H-R diagram, or YHGs have passed the red supergiant evolutionary stage.

Luminous Blue Variables (LBV) are massive evolved hot hypergiant stars ( $\left.T_{\text {eff }}>8000 \mathrm{~K}\right)$ showing 
spectroscopic and photometric variability reminiscent of cool hypergiants. The stellar mass of LBVs typically exceeds $40 \mathrm{M}_{\odot}$ and can in some cases be as large as $\sim 120 \mathrm{M}_{\odot} . \eta$ Car, P Cygni, and $\mathrm{S}$ Dor are examples of LBVs that have been frequently studied with high-resolution spectroscopy over the past half century. The LBVs are spectroscopically different from the Wolf-Rayet stars and their evolutionary phase is estimated not to exceed $\sim 50-100$ thousand years.

Hot and cool hypergiant stars exist near the Eddington luminosity limit, and exhibit a wide range of uncommon stellar properties. Their atmospheres are unstable. This causes quasi-periodic pulsation variability, strongly developed large-scale velocity fields, excessive mass-loss, and extended circumstellar envelopes. These extreme stars are possible progenitors of core collapse supernovae. An important property of hypergiants are recurring outbursts that signal the final stages in the fast evolution of the most massive stars. The outburst events occur on time-scales of decades to half a century revealing very large changes of $T_{\text {eff }}$ and commensurate strong spectral variability. For example, in 1999-2000, $\rho$ Cas decreased $T_{\text {eff }}$ from above $7000 \mathrm{~K}$ to below $4000 \mathrm{~K}$ during an outburst event that lasted $\sim 2$ years. Following the rapid $V$ brightness decrease of $\sim 1.3 \mathrm{mag}$, the YHG revealed TiO absorption bands in the near-IR spectrum which disappeared after returning to average $V$ brightness level. During the outburst, the mass-loss rate increased from $\sim 10^{-6} M_{\odot} y^{-1}$ to $5 \times 10^{-2} M_{\odot} y^{-1}$ (Lobel et al. 2003). LBVs reveal similar large (temporal) spectroscopic changes. During so-called $\mathrm{S}$ Doradus variability cycles, the $V$-brightness steadily increases over $\sim 10$ years while the spectrum transforms from being dominated by many prominent (metal) emission lines to strong absorption almost void of emission lines (e.g., S Dor and MWC 930, Lobel et al. 2017). The S Dor cycles are caused by stellar pulsations increasing $R_{*}$ and decreasing $T_{\text {eff }}$ to below 8000-9000 K.

Over the past 7 years, we performed long-term high-resolution spectroscopic monitoring of cool hypergiants, LBVs, and candidate LBVs (cLBV) with HERMES. All target stars are observed once or twice per semester for documenting long-term spectroscopic variability. However, our monitoring survey is currently limited to hypergiants with $V<13$ mag. Long-term spectroscopic monitoring of hypergiants is important for investigating if the short LBV phase is linked to binary evolution/formation (Martayan et al. 2016).

The study of hypergiants could benefit from spectroscopic observations with the 3.6-m DoT: - Low-resolution spectrograph (FosC): Spectra with a resolution of the order of $R \sim 4000$, as achievable with FOSC, will allow to extend our monitoring survey to include fainter targets such as the extragalactic hypergaints in M33. Long-term hypergiant monitoring with FosC is important for observing periodic flux variability of strong and broad Balmer $\mathrm{H}$ and $\mathrm{He}$ emission lines.

\subsection{Rapidly rotating A and B-type stars}

Y. Frémat (Yves.Fremat@oma.be) is interested in massive stars, especially those that rotate fast.

As shown in the left panel of Fig. 2, main sequence A- and B-type stars are known to rotate rapidly. In average their projected rotational velocity, $v \sin i$, is larger than $100 \mathrm{~km} \mathrm{~s}^{-1}$ and may even reach values close to the break-up velocity. Moreover, in this part of the HR diagram, it is expected that several stellar classes partly owe their peculiarities to rapid rotation (e.g. Be stars, He strong stars, $\delta$ Scuti stars, ...). Indeed, at such high rotation rates, the centrifugal forces expand the equator, giving the star a flattened shape, and generate surface non uniform temperature and density distributions that may impact its chemical composition and evolution. The temperature and density latitudinal gradients produce a gravitational darkening (GD) that introduces aspect angle effects into the spectra and colors. In some cases, these effects may be unambiguously detected at high SNR and high resolution in certain line profiles. This is the case, for example, in Vega, where depending on the formation region of the transition (e.g. equator, pole, or the complete surface) the line profiles may appear flattened (sometimes even reversed), triangular, or have the usual Voigt shape (Fig. 2, right panel). By 

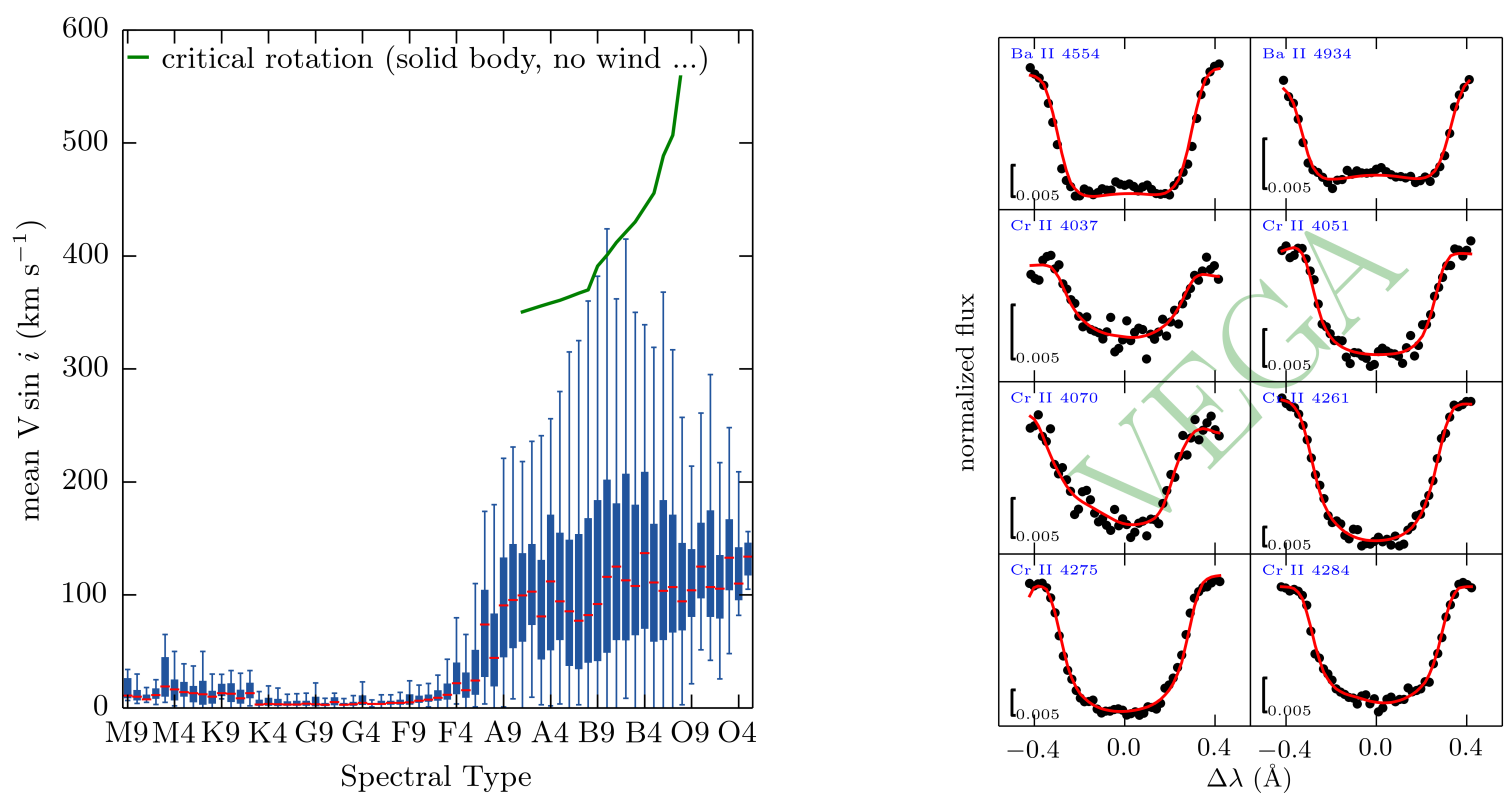

Figure 2: Left: Distribution of the mean projected rotational velocity and its spread as a function of the spectral type. The $v \sin i$ values are taken from the online catalogue of Głębocki \& Gnaciński (2005). The critical rotation rates (green curve) were computed with FASTROT (Frémat et al. 2005). Right: Line profiles affected by gravitational darkening in the spectrum of Vega (dots) fitted with theoretical line profiles (red curve) computed with FASTROT.

combining high-resolution with low-resolution spectra, we can therefore expect to be able to infer the inclination angle of the star, especially at lower $i$ values where the impact of GD on the spectrum is the most significant. On the other hand, the synthetic spectra used to characterize rapid rotators are often computed assuming rigid rotation, while there are clues for differential rotation to occur in Aand B-type stars. The departures from rigid rotation will have an impact on the temperature gradients as well as on the velocity fields observed at the surface. We aim to study the level of detectability of this impact by analysing simulations to be confronted to observations obtained both at high and low resolution in several open clusters. Our final goal is to study in detail the cluster distribution of the rotational velocities and of the inclination angles, and check how our findings and conclusions affect the interpretation of certain categories of rapid rotators (e.g. Be stars).

The science goals could be achieved with the 3.6-m DoT as soon as two instruments become available:

- High-resolution spectroscopy (HERMES-like): to obtain high-resolution spectra with SNR > 500) spectra for a detailed analysis. The astrophysical parameters and chemical abundances will be derived while the profiles of the weakest spectral lines will be studied assuming different rotation laws.

- Low-resolution spectrograph (FOSC): to obtain flux and wavelength calibrated spectra with a lower resolution covering the complete optical range $(350-900 \mathrm{~nm})$. It would be useful to extend the wavelength coverage of FOSC to $1100 \mathrm{~nm}$ and to match the spectral domain covered by the Gaia instruments. Moreover, the inclusion of two narrow-band filters around the Balmer jump would be very helpful to parameterize faint hot stars.

\subsection{Colliding winds in massive binaries}

R. Blomme (Ronny.Blomme@oma.be) is involved in the modelling of the effects of ocolliding winds as observed in massive binaries. 
Massive stars lose mass through a radiatively driven stellar wind. When two such stars form a binary, their stellar winds will collide, leading to interesting physical effects. The collision between the two winds heats up the material to such temperatures that it emits detectable X-ray radiation. In the shocks around the colliding-wind region, a fraction of the electrons are accelerated to high, nonthermal energies. These electrons spiral in the magnetic field, thereby emitting synchrotron radiation, which we can be detected at radio wavelengths. Furthermore, colliding winds also emit non-thermal $\mathrm{X}$-rays and gamma-rays, making these objects very relevant to high-energy physics. Frequently, these binaries have periods in the order of years.

The guaranteed access to the 3.6-m DoT telescope would be ideal for this project as soon as one instrument is offered:

- High-resolution spectroscopy (HERMES-like): It would make a long-term spectroscopic monitoring programme feasible. This would allow the determination of the orbital parameters of the longerperiod binaries. Such information is vital as input to the modelling of the wind-wind collision and predicting the resulting synchrotron and X-ray emission.

\subsection{Monitoring of post-AGB stars and planetary nebulae central stars}

The research of G. Van de Steene (G.Vandesteene@oma.be) focusses on the study of post-AGB stars and the central stars of planetary nebulae.

The final evolution of low- and intermediate-mass stars is a rapid transition from the Asymptotic Giant Branch (AGB) over the post-AGB transit towards the Planetary Nebula (PN) phase, before the stellar remnant cools down as a White Dwarf (WD). Arguably one of the most important issues which govern the discussion in the international research is the impact of binary interactions on stellar evolution. The presence of a companion can change the course of evolution and affects our understanding of the formation of PNe.

At Devasthal Observatory we would like to study the impact of binary interaction processes on the evolution of intermediate mass stars quantifying the orbital and chemical characteristics of post-AGB stars and PNe via longterm monitoring of their radial velocity and photometric variability. PostAGB photospheres bear witness of the total chemical changes induced by the drege-ups and binary interaction during the previous phases of evolution. The observed orbital information of post-AGB binaries can be used to deduce physical information of the system and the nature of the companions. From high-resolution spectra we can also determine the chemical composition and dynamics of the circumstellar material. Long term monitoring via high-resolution spectroscopy allows to investigate different types of objects and compose an evolutionary picture from the AGB to the PN stage which accounts for the morphological and chemical peculiarities, in combination with the orbital distribution and binarity rate.

The 3.6-m DOT would be ideal to study these faint objects provided that two types of instruments are available:

- Optical imager (FOSC): An optical imager allows the photometric monitoring of post-AGB stars and PN central stars to obtain information on their stellar pulsation and binarity.

- High-resolution spectroscopy (HERMES-like): More importantly, an efficient high-resolution spectrograph is needed to obtain accurate radial velocities to determine the orbital elements of the binary components, and to obtain chemical and dynamical information on the star and its circumstellar environment. 

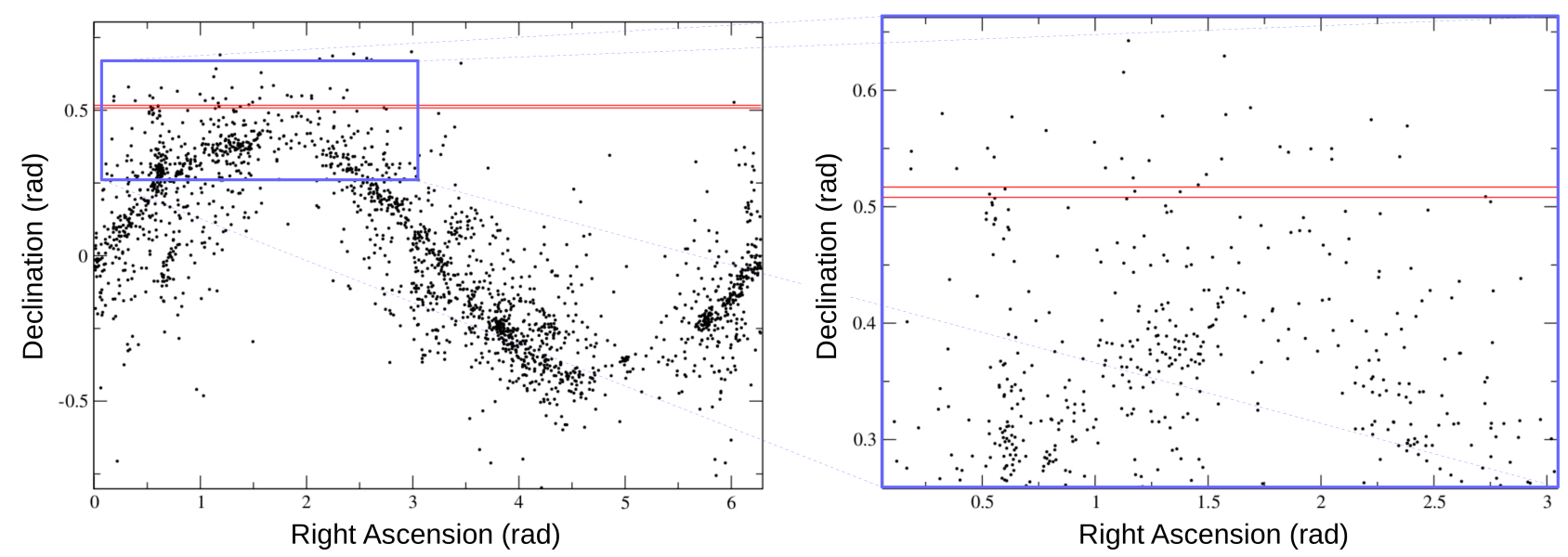

Figure 3: Location of known Trans-Neptunian Objects in the sky on April 1, 2017. The red lines show the scanning strip of the 4.0-m ILMT. The right panels zooms in on the targets that can be observed from the Devasthal Observatory.

\subsection{Revalorising the Ukkel Schmidt Telescope by Installing a CCD Camera (RUSTICCA)}

T. Pauwels (Thierry.Pauwels@oma.be) is mainly interested in small bodies of our Solar System.

The RUSTICCA project consisted in installing in 1995 a $3072 \times 2048$ CCD camera on the Ukkel Schmidt telescope, primarily to continue the astrometric observations of minor planets that started at Ukkel in 1924. The telescope has a main mirror of $1.2 \mathrm{~m}$, with a corrector plate of $0.85 \mathrm{~m}$. The focal length is $2.1 \mathrm{~m}$. With a pixel size of $9 \mu \mathrm{m}$, this gives a field of view of $45^{\prime} \times 30^{\prime}$. The limit magnitude is roughly 20. In the past 20 years, about 24000 astrometric positions of asteroids and comets have been produced with this camera, resulting in about 200 discoveries of minor planets. Also other observations have been performed: photometry of mutual phenomena of the Galilean satellites of Jupiter, occultation of stars by minor planets, photometry of mutual phenomena of an asteroid and its satellite and photometry of cataclysmic variables. Astrometry of asteroids have now come close to an end with the present equipment, considering the typical magnitude of still unknown objects, but we expect still to observe mutual phenomena and occultations in the future.

The ILMT data will contain, apart from stars, also Solar System objects. Main belt asteroids are in general rather fast and will remain only for a short period in the field of view of the ILMT, but Trans-Neptunian Objects (TNOs), with their slow motion in the sky, will remain observable for many months. With a limit magnitude of 23, there are still plenty of undiscovered TNOs in the range of the ILMT. The declination scanned is not optimal. With a latitude of 29.3 degrees, the scanning circle of the ILMT will not cross the ecliptic. Nevertheless, we expect at any moment between $0.2 \%$ and $0.5 \%$ of the total population of the TNOs to be in right declination range to come in the field of view of the ILMT (see Fig. 3 for the total population of TNOs in the sky on April 1, 2017). With the ecliptica culminating at a right ascension around $6 \mathrm{~h}(\sim 1.57 \mathrm{rad})$, we expect the months of November, December, January and February, when the ecliptic has its highest position in the sky at night, to be the most prolific for TNO discoveries. 


\section{Conclusions}

Several of the projects introduced in Section 2 could already be linked to the 4.0-m ILMT or to the first-generation instruments of the 3.6-m DOT. However, the science at the ROB would benefit the most from the BINA collaboration if an efficient high-resolution spectrograph similar to HERMES would be installed to the 3.6-m DoT too. If you are interested to collaborate with us, please contact P. De Cat (Peter.DeCat@oma.be) or the principle investigator of the project you are interested in (see Section 2).

\section{References}

Beaton R.L., Freedman W.L., Madore B.F. et al. 2016 ApJ, 832210

Briquet M., Aerts C. 2003, A\&A, 398, 687

De Jager C. 1998, A\&ARv 8, 145

Dupret M.-A., De Ridder J., De Cat P. et al. 2003, A\&A 398, 677

Frémat Y., Zorec J., Hubert A.-M., Floquet M. 2005, A\&A, 440, 305

Gamarova A. Yu., Mkrtichian D. E., Rodríguez E. et al. 2003, PASPC, 292, 369

Głębocki R., Gnaciński P. 2005, yCat, 3244, 0

Groenewegen M. A. T. 2013, A\&A 550, A70

Groenewegen M. A. T., Jurkovic M. I. 2017a, A\&A, 603, A70

Groenewegen M. A. T., Jurkovic M. I. 2017b, A\&A, 604, A29

Lampens P., Strigachev A., Kim S.-L. et al. 2011, A\&A 534, 111

Lampens P., Bognár Zs., Frémat Y. et al. 2015, EPJWC, 101, 6043

Lobel A., Dupree A.K., Stefanik R.P. et al. 2003, ApJ, 583, 923

Lobel A., Martayan C., Mehner A., Groh J.H. 2017, ASPC, 508, 245

Martayan C., Lobel A., Baade D. et al. 2016, A\&A, 587, A115

Naik M. B., Ojha D. K., Ghosh S. K. et al. 2012, BASI, 40, 531

Neiner C., Lampens P. 2015, MNRAS, 454, L86

Omar A., Yadav R. K. S., Shukla V. et al. 2012, Proceedings of the SPIE, 8446, 14

Pedicelli S., Lemasle B., Groenewegen M. A. T. et al. 2010, A\&A, 518, A18

Planck Collaboration, Aghanim N., Ashdown M., Aumont J. et al. 2016, A\&A, 596, A107

Raskin G., Van Winckel H., Hensberge H. et al. 2011, A\&A, 526, 69

Riess A. G., Macri L.M., Hoffmann S.L. et al. 2016, ApJ, 826, 56

Romaniello M., Primas F., Mottini M. et al. 2005, A\&A, 429, L37

Storm J., Gieren W., Fouqué P. et al. 2011, A\&A, 534, A95

Zima W. 2006, A\&A, 455, 227 